\title{
Estimation of frequency offset using warped discrete-Fourier transform
}

\author{
Ramji Venkataramanan ${ }^{\mathrm{a}, 1}$, K.M.M. Prabhu ${ }^{\mathrm{b}, *}$ \\ ${ }^{a}$ Department of EECS, University of Michigan, Ann Arbor, MI 48109-4122, USA \\ ${ }^{\mathrm{b}}$ Department of Electrical Engineering, Indian Institute of Technology, Madras, Chennai 600036, India
}

Received 17 January 2003; received in revised form 23 February 2005; accepted 11 May 2005

Available online 22 August 2005

\begin{abstract}
In this paper, the problem of estimating a small frequency offset in a signal with a large carrier frequency is addressed. The warped discrete-Fourier transform (WDFT) [A. Makur, S.K. Mitra, IEEE Trans. Circuits Systems-I: Fundam. Theory Appl. 6 (9) (September 2001) 1086-1093] is used and the accuracy of estimation and computational complexity of this technique is compared with the conventional discrete-Fourier transform (DFT) and the nonuniform discrete-Fourier transform (NDFT). A numerical example is provided to illustrate the comparison.
\end{abstract}

(C) 2005 Elsevier B.V. All rights reserved.

Keywords: DFT; NDFT; WDFT; FFT; Computational complexity

\section{Introduction}

There are many places (e.g., receivers and base stations of cellular systems) where a small frequency offset of a signal around a large carrier frequency needs to be estimated. Typically,

\footnotetext{
*Corresponding author. Tel.: +914422579368; fax: +914422570509 .

E-mail addresses: rvenkata@umich.edu

(R. Venkataramanan), prabhu_kmm@hotmail.com (K.M.M. Prabhu).

${ }^{1}$ Ramji Venkataramanan was at the Department of Electrical Engineering, Indian Institute of Technology, Madras. He is now a graduate student in the Department of EECS, Ann Arbor, USA.
}

a signal

$x(t)=A \cos \left(2 \pi\left(f_{\mathrm{c}}+\Delta f\right) t\right)$

is available for a finite length of time. It is sampled at a frequency $f_{\mathrm{s}}$ and converted into a discrete $\mathrm{N}$-point sequence which can be represented as

$x[n]=A \cos \left(\omega_{0}+\Delta \omega\right) n, \quad n=0,2, \ldots, N-1$,

where

$\omega_{0}=2 \pi \frac{f_{\mathrm{c}}}{f_{\mathrm{s}}}$ and $\Delta \omega=2 \pi \frac{\Delta f}{f_{\mathrm{s}}}$.

In this paper, the warped discrete-Fourier transform (WDFT) is used to estimate the offset $\Delta \omega$ given $x[n]$. In a related work [2], single 
frequency estimation using WDFT has been discussed.

Our paper is organized as follows: The WDFT and the nonuniform discrete-Fourier transform (NDFT) are briefly explained in this section. In Section 2, the application of the WDFT to estimate frequency offsets is discussed. The computational procedure is described and an example is provided. In Section 3, the results of the WDFT technique are compared with the discrete Fourier transform (DFT) and the NDFT on the basis of accuracy and computational complexity. Conclusions are presented in Section 4.

\subsection{The discrete-Fourier transform (DFT)}

The DFT finds widespread applications in the spectral analysis of discrete-time signals. The $N$ point DFT, $X[k], 0 \leqslant k \leqslant N-1$, of a length $N$ sequence $x[n]$ is given by the frequency samples of the $z$-transform, $X(z)$ of $x[n]$, evaluated at $N$ uniformly spaced points on the unit circle, i.e.,

$$
\begin{gathered}
X[k]=\sum_{n=0}^{N-1} x[n] \mathrm{e}^{-\mathrm{j} 2 \pi k / N}, \\
0 \leqslant k \leqslant N-1 .
\end{gathered}
$$

In the problem of frequency offset estimation, it is desirable to compute the frequency samples of $x[n]$ only at frequencies close to $\omega_{0}$. Owing to the fixed frequency resolution of $2 \pi / N$, the length of the DFT to estimate small $\Delta \omega$ is very large. So the use of alternative techniques, such as the warped DFT [1] and the NDFT [3] is considered.

\subsection{The nonuniform discrete-Fourier transform (NDFT)}

The NDFT [3] of an $N$ point sequence $x[n]$ is defined as

$X_{\mathrm{ND}}[k]=X\left(z_{k}\right)=\sum_{n=0}^{N-1} x[n] z_{k}^{-n}, \quad 0 \leqslant k \leqslant N-1$.

The NDFT, the most general form of the DFT, evaluates the frequency samples of $X(z)$ at $N$ arbitrary, distinct points on the $z$-plane. In matrix form, the equations can be written as

$$
\begin{aligned}
& {\left[X_{\mathrm{ND}}[0], \ldots, X_{\mathrm{ND}}[N-1]\right]^{\mathrm{T}}} \\
& \quad=\boldsymbol{D}_{N}[x[0], \ldots, x[N-1]]^{\mathrm{T}},
\end{aligned}
$$

where $\mathbf{D}_{N}$ is the $N \times N$ NDFT matrix given by

$$
\mathbf{D}_{N}=\left[\begin{array}{ccccc}
1 & z_{0}^{-1} & z_{0}^{-2} & . . & z_{0}^{-(N-1)} \\
1 & z_{1}^{-1} & . . & . . & z_{1}^{-(N-1)} \\
1 & . . & . . & . . & . . \\
1 & . . & . . & . . & . . \\
1 & z_{N-1}^{-1} & . . & . . & z_{N-1}^{-(N-1)}
\end{array}\right] .
$$

For our problem of estimating frequency offset, the NDFT can be used to evaluate the frequency samples $X\left(\mathrm{e}^{\mathrm{j} \omega}\right)$ at $\omega$ close to $\omega_{0}$ and thereby estimate the offset. In general, the computation of the NDFT samples involves the multiplication of $\mathbf{D}_{N}$ with the length $N$ vector composed of samples of $x[n]$. The NDFT offers complete flexibility in choosing the sampling points which leads to spectral resolution that can be controlled as desired. However, in general it is computationally intensive, requiring $N^{2}$ complex multiplications for the direct method. In some special cases, such as when the NDFT is evaluated at points on the unit circle, a reduction in computational complexity can be achieved by using methods such as the Goertzel algorithm [4].

\subsection{The warped discrete-Fourier transform (WDFT)}

The WDFT, proposed recently [1], is a special case of the NDFT. Here, the locations of the frequency points are modified by applying an allpass transformation to warp the frequency axis. Then, uniformly spaced points on the warped frequency axis are equivalent to nonuniformly spaced points on the original frequency axis. By choosing the warping parameters suitably, we can place some of the frequency samples close to each other to provide higher resolution in the frequency range of interest without increasing the length of the DFT.

The WDFT of a sequence $x[n]$ is defined as follows. We first choose a polynomial $A\left(z^{\prime}\right)$ to construct an allpass function. Let $A\left(z^{\prime}\right)$ be a 
polynomial in $z^{\prime}$ with degree $M$. Define

$X\left(z^{\prime}\right)=\sum_{n=0}^{N-1} x[n]\left[\frac{A\left(z^{\prime}\right)}{z^{\prime-M} A\left(z^{\prime-1}\right)}\right]=\frac{N\left(z^{\prime}\right)}{D\left(z^{\prime}\right)}$.

The $N$ point WDFT $X_{W}[k]$ of $x[n]$ consists of samples of $X\left(z^{\prime}\right)$ evaluated at $\mathrm{e}^{\mathrm{j} 2 \pi k / N}$, i.e.,

$X_{W}[k]=\left.X\left(z^{\prime}\right)\right|_{\mathrm{e}^{\mathrm{j} 2 \pi k / N}}=\frac{\left.N\left(z^{\prime}\right)\right|_{\mathrm{e}^{2} 2 \pi k / N}}{\left.D\left(z^{\prime}\right)\right|_{\mathrm{e}^{\mathrm{j} 2 \pi k / N}}}, 0 \leqslant k \leqslant N-1$.

The reader is referred to [1] for a detailed description of the WDFT.

\section{Application of WDFT to estimate frequency offset}

For efficient estimation of the frequency offset $\Delta \omega$ in (2), the frequency response of $x[n]-X\left(e^{\mathrm{j} \omega}\right)$ has to be sampled densely at frequencies close to $\omega_{0}$. The frequency sample that gives the maximum value of $\left|X\left(\mathrm{e}^{\mathrm{j} \omega}\right)\right|$ enables us to determine the frequency offset around $\omega_{0}$. The parameters that have to be chosen are:

1. the length of the WDFT used,

2. the order $M$ of the allpass function $C\left(z^{\prime}\right)$,

3. the coefficients of $A\left(z^{\prime}\right)$.

Assuming the parameters have been chosen, the computational procedure we use for efficiently computing the WDFT is described below.

\subsection{Computation of the WDFT}

We use the matrix-based implementation proposed in [1] for the computation of the WDFT. We give a very brief outline of the computational procedure here; the details are described in [1]. Suppose we want to compute the $N$ point WDFT of $x[n]$. Define

$N_{1}\left(z^{\prime}\right)=N\left(z^{\prime}\right) \bmod z^{\prime N}$

with $N\left(z^{\prime}\right)$ as in (7). $N_{1}\left(z^{\prime}\right)$ is a polynomial of degree $N-1$. Similarly, define

$D_{1}\left(z^{\prime}\right)=D\left(z^{\prime}\right) \bmod z^{\prime N}$.
Then, the WDFT (8) can be computed by replacing $N\left(z^{\prime}\right)$ and $D\left(z^{\prime}\right)$ with $N_{1}\left(z^{\prime}\right)$ and $D_{1}\left(z^{\prime}\right)$ respectively. That is,

$$
X_{W}[k]=\left.X\left(z^{\prime}\right)\right|_{z^{\prime}=\mathrm{e}^{\mathrm{i} 2 \pi k / N}}=\frac{\left.N_{1}\left(z^{\prime}\right)\right|_{z^{\prime}=\mathrm{e}^{\mathrm{j} 2 \pi k / N}}}{\left.D_{1}\left(z^{\prime}\right)\right|_{z^{\prime}=\mathrm{e}^{\mathrm{i} 2 \pi k / N}}},
$$$$
0 \leqslant k \leqslant N-1 \text {. }
$$

It is shown in [1] that this can be efficiently computed for a given signal $x[n], 0 \leqslant n \leqslant N-1$, denoted $\mathbf{x}$ in vector form. In particular, it is shown that

$\left[X_{W}[0] X_{W}[1], \ldots, X_{W}[N-1]\right]^{\mathrm{T}}=\Lambda_{D} \cdot \mathbf{W} \cdot \mathbf{Q} \cdot \mathbf{x}$,

where $\mathbf{W}$ is the standard $N \times N$ DFT matrix, $\mathbf{Q}$ is an $N \times N$ real matrix [1] and

$$
\boldsymbol{\Lambda}_{D}=\left[\begin{array}{ccccc}
\frac{1}{D_{1}[0]} & 0 & 0 & \ldots & 0 \\
0 & \frac{1}{D_{1}[1]} & \ldots & . . & 0 \\
\cdot & & . & . . & \\
\cdot & & & . & \\
0 & 0 & & & \frac{1}{D_{1}[N-1]}
\end{array}\right] .
$$

We now present an example to illustrate the procedure and compare the results obtained with the WDFT to those obtained with the DFT and the NDFT.

\subsection{Example}

In (1), let $f_{\mathrm{c}}$ and $f_{\mathrm{s}}$ be such that $f_{\mathrm{s}}=4 f_{\mathrm{c}}$ and so $\omega_{0}=(\pi / 2)$. Further, let the sequence $x[n]$ be of length 64 , i.e., $N=64$. When an $N$-point DFT is used, the frequency separation between adjacent samples is $(2 \pi / N) \mathrm{rad} /$ sample.

So, the maximum offset estimation error using a 64-point DFT is

$\operatorname{err}_{\mathrm{DFT}}=\frac{1}{2} \times \frac{2 \pi}{64}=0.049 \mathrm{rad} /$ sample.

If we assume that $f_{\mathrm{c}}=10 \mathrm{KHz}$, then this means that a 64-point DFT can estimate frequency offsets around $f_{\mathrm{c}}$, with a maximum error of

$\Delta f_{\mathrm{DFT}}=\operatorname{err}_{\mathrm{DFT}} \times \frac{f_{\mathrm{s}}}{2 \pi}=312.5 \mathrm{~Hz}$.

We now consider a 64-point WDFT with $C\left(z^{\prime}\right)$, a second-order allpass function $(M=2)$. 
Let $A\left(z^{\prime}\right)$ be given by $z^{\prime-2}+a z^{-1}+b$. Then the frequency mapping is given by

$z^{-1}=C\left(z^{\prime}\right)=\frac{z^{\prime-2}+a z^{\prime-1}+b}{b z^{\prime-2}+a z^{\prime-1}+1}$,

where $a$ and $b$ are real coefficients to be chosen suitably.

Substituting $z=\mathrm{e}^{\mathrm{j} \omega}$ and $z^{\prime}=\mathrm{e}^{\mathrm{j} \omega^{\prime}}$, we obtain

$\mathrm{e}^{-\mathrm{j} \omega}=\frac{\mathrm{e}^{-\mathrm{j} 2 \omega^{\prime}}+a \mathrm{e}^{-\mathrm{j} \omega^{\prime}}+b}{b \mathrm{e}^{-\mathrm{j} 2 \omega^{\prime}}+a \mathrm{e}^{-\mathrm{j} \omega^{\prime}}+1}=\frac{A\left(\mathrm{e}^{\mathrm{j} \omega^{\prime}}\right)}{A^{\prime}\left(\mathrm{e}^{\mathrm{j} \omega^{\prime}}\right)}$.

Using

$A^{\prime}\left(\mathrm{e}^{\mathrm{j} \omega^{\prime}}\right)=\mathrm{e}^{-\mathrm{j} 2 \omega^{\prime}} A\left(\mathrm{e}^{-\mathrm{j} \omega \omega^{\prime}}\right)$

and

$A^{\prime *}\left(\mathrm{e}^{\mathrm{j} \omega^{\prime}}\right)=A^{\prime}\left(\mathrm{e}^{-\mathrm{j} \omega^{\prime}}\right)$,

we obtain the frequency mapping by equating the real and imaginary parts on either side of (17) as

$\cot \left(\frac{\omega}{2}\right)=\frac{a \operatorname{cosec}\left(\omega^{\prime}\right)+(1+b) \cot \left(\omega^{\prime}\right)}{1-b}$.

\section{Comparison of different algorithms}

We now compare the performance of the WDFT with two other techniques, viz., the simple DFT and the DTFT sampled in the frequency range of interest (NDFT). The algorithms are evaluated on the basis of accuracy of determining the frequency offset and the computational complexity involved. For easy comparison, we use the simple example considered in the previous section.

\subsection{Accuracy}

We propose evaluating the WDFT with 2 sets of values for the pair $[a, b]$ - one set of values for frequency points corresponding to positive deviations around $\pi / 2$ and the other for negative deviations. This was found to give better resolution than a single WDFT spanning frequency samples on either side of $(\pi / 2)$. Equivalently, a much larger length of WDFT is required to get a resolution comparable to that obtained by using two appropriate sets of values for $a$ and $b$. For a 64-point WDFT, using $[a=2.176, b=-1.166]$ and $[a=-2.087, b=-1.633]$ was found to give the best resolution for $\omega$ around $\pi / 2$ for negative and positive deviations, respectively. The frequency mapping for $[a=2.176, b=-1.166]$ is shown in Fig. 1. The corresponding frequency points $\omega$ on the $z$-plane are as shown in Fig. 2 .

The 64-point WDFT was computed for each of the sets of $[a, b]$ using (12). The offset was determined using (20) from the frequency at which the WDFT sample had the largest magnitude. The performance of this method was tested on MATLAB using offsets $\Delta \omega$ around $\pi / 2$ ranging from $-0.05 \mathrm{rad} / \mathrm{sample}$ to $+0.05 \mathrm{rad} / \mathrm{sample}$. The estimated offset for a 64-point DFT and 64-point WDFT (evaluated at 2 sets of values of $[a, b]$ ) are shown in Fig. 3. The offset estimation error for each case is shown in Fig. 4. As indicated by (14), a 64-point DFT cannot estimate offsets in the specified range. In comparison, a 64-point WDFT can estimate offsets in the range with a maximum error of

$\operatorname{err}_{\mathrm{WDFT}}=0.0055 \mathrm{rad} / \mathrm{sample}$.

For the $f_{\mathrm{c}}$ and $f_{\mathrm{s}}$ used in (15), this translates to

$\Delta f_{\mathrm{WDFT}}=\operatorname{err}_{\mathrm{WDFT}} \times \frac{f_{\mathrm{s}}}{2 \pi}=35 \mathrm{~Hz}$.

As shown in Fig. 4, to get a performance to the comparable 64-point WDFT $(\times 2)$, a 1024-point DFT is required.

We now examine the performance obtained by using a 64-point NDFT. The frequency sample points are chosen to be uniformly spaced in the frequency range of interest $[\pi / 2-0.05 \mathrm{rad}$, $\pi / 2+0.05 \mathrm{rad}]$. Thus, this is the DTFT sampled at 64 points spaced in $[\pi / 2-0.05 \mathrm{rad}$, $\pi / 2+0.05 \mathrm{rad}]$. The maximum error obtained is

$\operatorname{err}_{\mathrm{NDFT}}=0.0016 \mathrm{rad} / \mathrm{sample}$

and

$\Delta f_{\mathrm{NDFT}}=\operatorname{err}_{\mathrm{NDFT}} \times \frac{f_{\mathrm{s}}}{2 \pi}=10.2 \mathrm{~Hz}$.

Evidently, the NDFT offers better resolution than the WDFT for $M=2$. However, this is achieved at the expense of an increase in the number of computations, as discussed in the next section. It should also be noted that by using a higher-order warping function (larger $M$ ) and 
choosing the warping parameters suitably, the resolution of the WDFT can be improved significantly.

\subsection{Computational complexity}

Let $\mathbf{x}$ be the $N$-dimensional input vector (complex in general). An $N$-point DFT requires $(\mathrm{N} / 2) \log _{2} N$ complex multiplications and $N \log _{2} N$ complex additions. Since one complex multiplication involves four real multiplications and two real additions, and one complex addition involves two real additions, an $N$-point FFT involves $2 N \log _{2} N$ real multiplications and $3 N \log _{2} N$ real additions.

Evaluation of the WDFT as a product of matrices involves $N\left(N+2 \log _{2} N+4\right)$ real multiplications and $N\left(2 N+3 \log _{2} N\right)$ real additions [1]. For $N=64$, this works out to 5120 real multiplications and 9344 real additions. For an equivalent performance, a 1024-point FFT is required which involves 20,480 real multiplications and 30,720 real additions.

If we use the NDFT, we get maximum flexibility in choosing the sample points. The resolution can be controlled more precisely than the WDFT, but at the expense of increased computation. The direct method of evaluating of the NDFT involves $4 N^{2}$ real multiplications and $N(4 N-2)$ real addi-

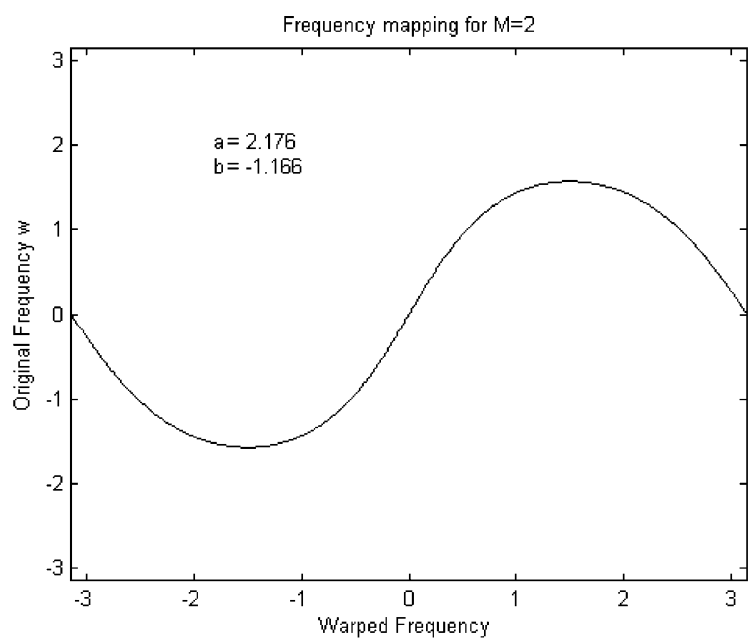

Fig. 1. Frequency mapping for second-order allpass warping function $(M=2)$.
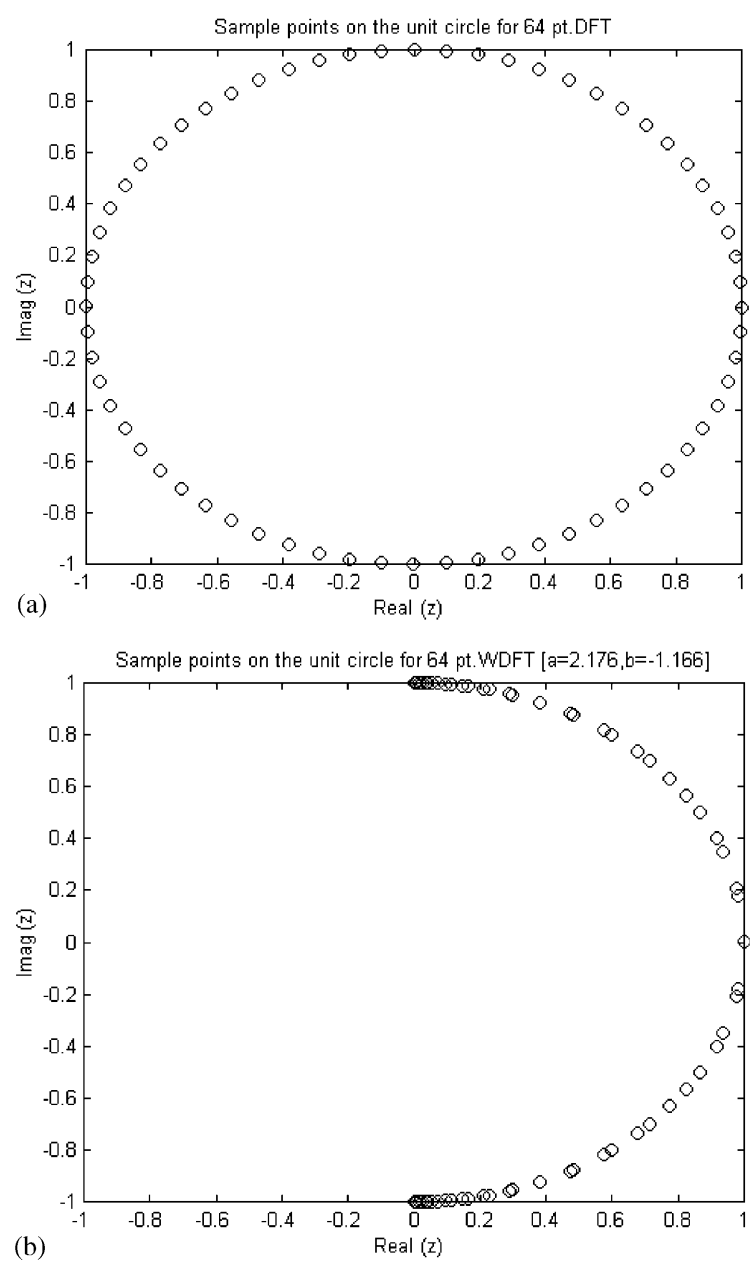

Fig. 2. Frequency sample points on the $Z$-plane.

tions. For $N=64$, this works out to 16,384 real multiplications and 16,256 real additions. Using the fact that the NDFT is being evaluated at points on the unit circle in the $z$-plane, the number of computations can be reduced using the Goertzel algorithm [4]. The second-order recursive filter implementation of the Goertzel algorithm requires $N(2 N+8)$ real multiplications and $N(4 N+6)$ real additions. For $N=64$, this works out to 8704 real multiplications and 16,768 real additions.

The complexities of the different algorithms for $N=64$ are summarized in Table 1. When larger values of $N$ are required, the computational savings obtained by the WDFT is still higher. 


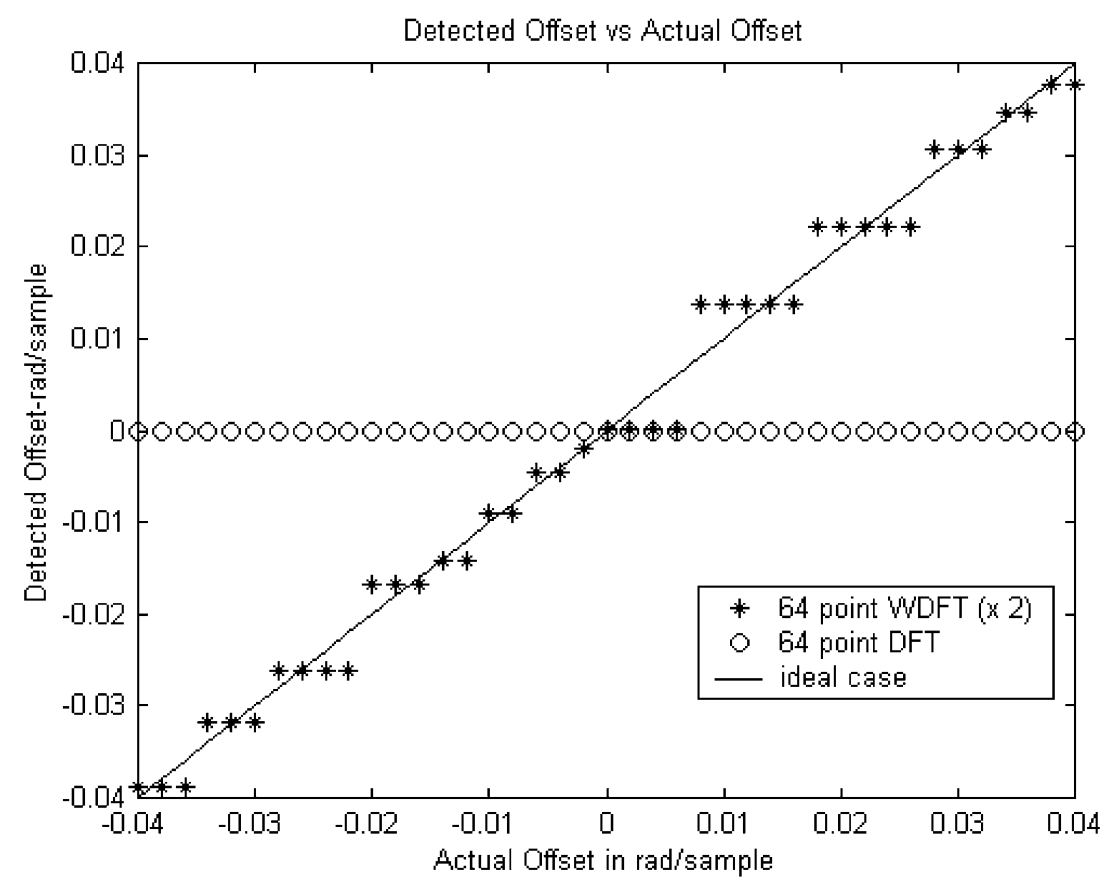

Fig. 3. Frequency offset, as estimated by DFT and WDFT.

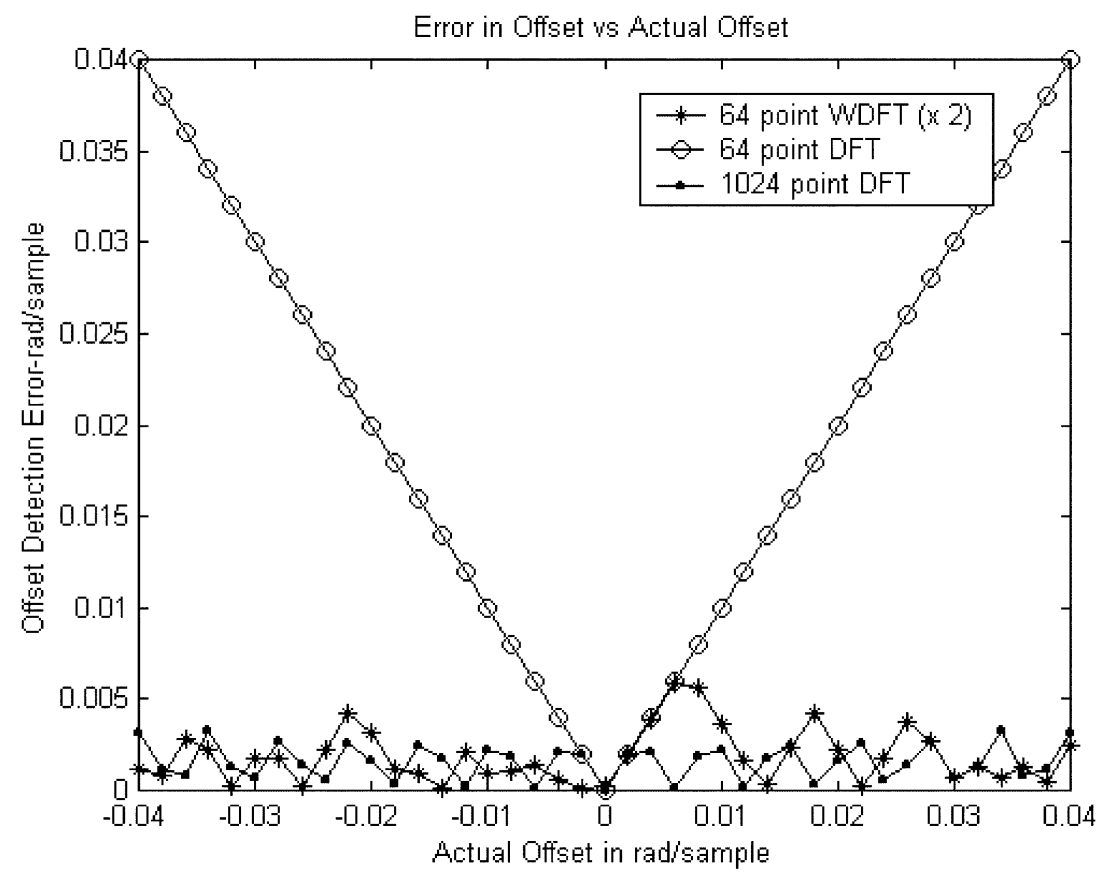

Fig. 4. Error in frequency offset, as estimated by DFT and WDFT. 
Table 1

Computational complexities of different algorithms

\begin{tabular}{lcc}
\hline Algorithm $(N=64))$ & \multicolumn{2}{l}{ Computations } \\
\cline { 2 - 3 } & $\begin{array}{l}\text { Number of } \\
\text { multiplications }\end{array}$ & $\begin{array}{l}\text { Number of } \\
\text { additions }\end{array}$ \\
\hline DFT & 768 & 1152 \\
WDFT & 5120 & 9344 \\
Nonuniformly sampled & 16,384 & 16,256 \\
DTFT (Goertzel algorithm) & & \\
\hline
\end{tabular}

\section{Conclusions}

In this paper, we discuss the application of the WDFT to estimate frequency offsets. We have shown that the WDFT, with parameters appropriately chosen, gives superior performance in terms of resolution and computational complexity over the FFT. It is also computationally more efficient than the sampled DTFT method (NDFT), especially for large values of $N$, although the latter offers more flexibility in choosing the frequency sample points. Though the example presented in this paper uses a second-order allpass function for warping, a higher-order function could be easily used. This increases the number of parameters to be chosen, which would lead to better resolution by offering more flexibility in choosing the sampling points.

\section{References}

[1] A. Makur, S.K. Mitra, Warped discrete-Fourier transform: theory and applications, IEEE Trans. Circuits Systems-I.: Fundam. Theory Appl. 6 (9) (September 2001) 1086-1093.

[2] S. Franz, G. Doblinger, S.K. Mitra, Frequency estimation using warped discrete Fourier transform, Signal Processing 83 (2003) 1661-1671.

[3] S. Bagchi, S.K. Mitra, The nonuniform discrete Fourier transform and its applications in filter design: Part I-1-D, IEEE Trans. Circuits Systems-II: Analog Digital Signal Processing 43 (6) (June 1996) 422-433.

[4] S. Bagchi, S.K. Mitra, The Nonuniform Discrete Fourier Transform and its Applications in Signal Processing, Kluwer Academic Publishers, Dordrecht, 1999. 